\title{
Properties and applications of FRP in strengthening RC structures: a review
}

\begin{abstract}
In civil and structural engineering, building structures with robust stability and durability using sustainable materials is challenging. The current technological means and materials cannot decrease weight, enlarge spans, or construct slender structures, thus inspiring the exploration for valuable composite materials. Fiber reinforced polymer (FRP) features high-strength and lightweight properties. Using FRP motivates civil engineers to strengthen existing RC structures and repair any deterioration. With FRP, a system that can resist natural disasters, such as earthquakes, strong storms, and floods, can be developed. However, deterioration of structures has become a critical issue in modern construction industries worldwide. This paper reviews the FRP design, matrix, material properties, applications, and serviceability performance. This literature review also aims to provide a comprehensive insight into the integrated applications of FRP composite materials for improving the techniques of rehabilitation, comprising the applications toward the repair, strengthening, and retrofit of concrete structures in the construction industry today.
\end{abstract}

Keyword: Applications; Aramid/Basalt/Glass/Carbon fiber reinforced polymer (AFRP, BFRP, GFRP, CFRP) beams; Columns; Deterioration; Fiber reinforced polymer (FRP); Joints; Matrix; Properties; Rehabilitation; Reinforced concrete structures; Serviceability and strengthening structures 